\title{
Arbor
}

\section{Racionalidad y Toma de Decisiones hacia el siglo XXI}

Sixto Ríos

Arbor CLXIII, 643-644 (Julio-Agosto 1999), 437-467 pp.

Tratamos de reflejar en este artículo el estado actual, desarrollo, tensiones, actividades investigadoras y problemas abiertos al futuro prometedor de esta actividad científico-práctica ilustrando nuestra exposición con ejemplos en parte tomados de trabajos realizados sucesivamente con nuestros discípulos y colaboradores de la Escuela de Estadística de la Universidad Complutense, del Departamento de I.O. de la Universidad Complutense, del Instituto de Investigación Operativa y Estadística (CSIC), de Bedaux, SOFEMASA, METRA-6, del Grupo de Análisis de decisiones de la Real Academia de Ciencias en nuestra etapa final de trabajo.

Está claro que si no queremos que el público pierda la confianza en el proceso asociado al control del riesgo por los científicos, los tecnólogos y administradores se han de tomar con el mayor rigor las decisiones que pueden influir en el futuro bienestar de todos; es decir, se debe aprender a tratar el riesgo y la incertidumbre en las decisiones importantes individuales o colectivas.

Puede decirse que el hombre se ha preocupado del problema de tomar buenas decisiones al menos desde los tiempos de la República de Platón, obra que se considera el origen remoto de muchos de- 
sarrollos y progresos posteriores, que han conducido tras un largo proceso científico-cultural a los fecundos métodos actuales de toma de decisión (T.D.) es tan importantes en el marco de la cultura occidental. Pero es frecuente encontrar personas cultas que creen que no es posible utilizar hoy métodos racionales, científicamente elaborados, para tomar decisiones por uno o por varios individuos en situaciones de incertidumbre, complejas e importantes, que reflejen sus informaciones e intereses. Así los problemas de un "equipo" que ha de llegar a una decisión global para la ubicación de una red de aeropuertos para un país o de una constelación de hospitales para una ciudad o a la localización optima de un conjunto de depósitos de residuos radioactivos para una Comunidad de Estados,... Las diferencias de costes y beneficios de unas o otras soluciones son a veces enormes y hay que tener especialmente en cuenta las consecuencias de una mala solución sobre todo en los problemas que implican riesgos para el medio ambiente y la salud humana y de otros seres vivos, etc. Sin esta preparación racional básica seria de la T. D. resultan a veces vacías de su sentido las "discusiones políticas", de una tertulia, de un parlamento, de un gobierno, de un cuerpo electoral,...

En esta clara tendencia de nuestra sociedad actual al crecimiento del desarrollo científico y tecnológico y a su utilización para la mejora de nuestras vidas nos encontramos cada día con enormes incertidumbres sobre el futuro lleno de nuevos riesgos que debemos afrontar. Si ayer se rompió una presa con gran cantidad de residuos contaminados y mañana establecemos un nuevo depósito de residuos químicos o nucleares, creamos nuevas fuentes de contaminación de agua y atmósfera, cuya influencia negativa en nuestra salud debemos medir y controlar en lo posible tal riesgo e incertidumbre.

Estos objetivos de compaginar el desarrollo tecnológico con un control apropiado que permita mejorar nuestras vidas en vez de empeorarlas, constituyen horizontes que se pueden realizar gracias al desarrollo creciente actual del Análisis de decisiones.

Es poco frecuente que personas de nuestro tiempo den importancia a las votaciones. No piensan quizá que son la base de las decisiones colectivas cuyos resultados influirán en nuestras vidas y las de nuestros descendientes durante largos periodos de tiempo.

Los sistemas democráticos vienen rodando desde los griegos o quizá antes y no es posible olvidar la gravemente famosa votación del jurado en el juicio a Sócrates (339 a. J. C.): el proceso de decisión estableció dos etapas, $1^{\mathrm{a}}$ ) votación: culpable / no culpable; $2^{\mathrm{a}}$ votación: si culpable, 


\section{Racionalidad y toma de decisiones hacia el siglo XXI}

qué pena se le impone. Los resultados fueron: $1^{\mathrm{a}}$ ) culpable por 280 votos contra $\left.221 ; 2^{a}\right) 360$ votos a favor de la pena de muerte y 141 en contra. Este resultado perverso, en que muchos jurados que, en la primera votación afirmaron que Sócrates era inocente, votaron en la segunda por que tomara la cicuta, pone de relieve las dificultades de la racionalidad colectiva.

Y el autor de estas líneas no ha podido olvidar, después de casi 50 años, la decisión que tuvo que tomar en Mérida (Venezuela) tras un intento de viajar en avión a Caracas, intento fallido por paro de los dos motores del avión y aterrizaje forzoso en un pantano de lodo, sin heridos graves en el interior del avión, pero muerte de una niña que paseaba tranquilamente por el campo. Aquel día el autor, entonces experto de la UNESCO y Director de la Escuela de Estadística de la Universidad central de Venezuela debía tomar una decisión para intentar nuevamente regresar a Caracas para lo que había dos alternativas posibles: a) tomar otro avión análogo en el que probablemente vigilarían más el vuelo y los aviadores por el bien de la compañía y de los viajeros o bien b) tomar un taxi en que debía pasar unas diez horas atravesando los Andes por puertos y vericuetos, con conductores dudosos. Este es el tipo de problemas que se llaman en incertidumbre total o ignorancia, en que las matemáticas que entonces se conocían podían ayudar poco, pero al menos tuvimos la "otra ayuda" que nos permite hoy contarlo como una anécdota.

Tratamos de reflejar en este articulo el estado actual, desarrollo, tensiones, actividades investigadoras y problemas abiertos al futuro prometedor de esta actividad científico-práctica ilustrando nuestra exposición con ejemplos en parte tomados de trabajos realizados sucesivamente con nuestros discípulos y colaboradores de la Escuela de Estadística de la U. Complutense, del Departamento de I.O. de la Universidad Complutense, del Instituto de Investigación Operativa y Estadística (CSIC), de Bedaux, SOFEMASA, METRA-6, del Grupo de Análisis de decisiones de la Real Academia de Ciencias en nuestra etapa final de trabajo,...

Esta claro que si no queremos que el público pierda la confianza en el proceso asociado al control del riesgo por los científicos, los tecnólogos y administradores se han de tomar con el mayor rigor las decisiones que pueden influir en el futuro bienestar de todos; es decir, se debe aprender a tratar el riesgo y la incertidumbre en las decisiones importantes individuales o colectivas.

En mas de una ocasión se ha dicho que se trata de una línea de investigación científica aplicable en que más que en cualquier otra, se han ob- 


\section{Sixto Ríos}

tenido los mejores dividendos con modestos recursos, como buenos ordenadores y mejores células grises.

\section{Decisiones en certidumbre}

Un primer campo importante es el estudio de las decisiones individuales en problemas en que se suponen conocidos con certidumbre los resultados finales de las alternativas posibles, y tales resultados se consideran medibles con criterio único bien definido. Su estudio condujo durante las décadas 1950-60 a la Investigación Operativa (I.O.), conjunto de poderosas metodologías de optimización, llamadas programación lineal y no lineal, programación paramétrica, dinámica, etc. El desarrollo paralelo del ordenador en dichos años, permitió la resolución numérica efectiva de problemas deterministas y monocriterio, de gran complejidad, especialmente originados en las necesidades surgidas al enfrentarse grupos de científicos sobresalientes con las dificultades asociadas a la dirección y manejo táctico y estratégico de los ejércitos, en la II Guerra Mundial.

Esencialmente en todos estos problemas el modelo matemático o representación matemática simplificada de la realidad, consiste en construir y optimizar una función objetivo $\mathrm{f}(\mathrm{x})$, en que $\mathrm{x}$ es una variable de decisión que representa los factores que el decisor puede controlar dentro de un conjunto de restricciones $\mathrm{x} \in \mathrm{C}$ que definen y limitan sus posibilidades. P.e. en el famoso problema del viajante se trata de encontrar el camino más corto para salir de Madrid y volver al punto de partida recorriendo todas las capitales de provincia haciendo el viaje en tiempo mínimo. En este ejemplo, la función objetivo a minimizar es la suma de tiempos $\mathrm{f}(\mathrm{t})=\sum \mathrm{t}_{\mathrm{i}}$, y la solución general de tal problema resultó difícil durante algunos años.

\section{Decisiones en incertidumbre}

Pero aunque algunos investigadores operativos creyeron que habían resuelto los principales problemas de la optimización, pronto se advirtió que cuando en las consecuencias interviene el azar o la incertidumbre, o múltiples criterios o varios decisores, etc., se hace necesario introducir nuevas ideas, y es entonces cuando comienza propiamente la Teoría de la Decisión que, justamente por estas características peculiares, se diferencia de la Teoría clásica de la Programación y Optimización. 


\section{Racionalidad y toma de decisiones hacia el siglo XXI}

Las decisiones más importantes de la empresa, de la administración, de la ecología, de la medicina, de la justicia, implican, en efecto, un porvenir incierto. La investigación, la creatividad, el desarrollo, tienen consecuencias cuya característica principal es la incertidumbre. Se hace, pues, preciso aprender a tomar decisiones en presencia de una incertidumbre, irreducible por su misma esencia y, por tanto, habrá necesidad de intentar medirla y saber que riesgos comportan nuestras decisiones.

Tales medidas no podrán ni deberán evitar una componente subjetiva, que permita tener en cuenta las creencias y las preferencias personales de los interesados en relación con la situación de decisión y sus resultados posibles.

El estudio científico de modelos y métodos para tomar decisiones optimas o, al menos, satisfactorias, constituye la Ciencia o Teoría de la Decisión. Y el Análisis de Decisiones abarca un conjunto de metodologías que permiten transformar problemas complejos de decisión en sucesiones de otros sencillos y transparentes, relacionándolos entre sí mediante reglas 0 axiomas de manera lógica y comprensible.

Con esta estrategia del "divide y vencerás", pretendemos llegar en cada problema complejo a tener una visión global de su estructura, a través de representaciones formales como árboles de decisión, diagramas de influencia, sistemas expertos, en las que se integren las percepciones probabilísticas del decisor en relación con los sucesos inciertos que se le presentan, sus valoraciones de las consecuencias de las posibles decisiones para llegar a la aplicación de criterios normativos y fundamentales que permitan ofrecer a los decisores soluciones racionales coherentes y en consonancia con su pensamiento critico y comportamiento natural. Así se hace compatible la racionalidad científica con la inevitable y aun deseable presencia de lo subjetivo.

Los trabajos de V. Neumann-Morgenstern (1943) son el punto de partida del tratamiento científico moderno de los problemas de decisión individual y también de las decisiones en concurrencia: juegos de estrategia, negociaciones,... Las investigaciones de Arrow son paralelamente, a partir de 1951, el origen del estudio de los problemas de las decisiones colectivas fundamentales en la vida política de las sociedades modernas. Una idea de la importancia del tema se tiene al considerar que al menos doce Premios Nobel: Arrow, Debreu, Kantorovich, Koopmans, Allais, Simon, Markowitz, Nash, Selten, Harsany, Black, Scholes, Sen,... han dedicado un buena parte de sus investigaciones a los problemas de las decisiones humanas, cuya versatilidad inagotable ha hecho necesarios los esfuerzos de matemáticos, estadísticos, informáticos, psicólogos, economistas, ingenieros, militares, médicos, abogados, politólogos, decisiólogos,..., para ir construyendo año tras año una sucesión de metodologías cada vez mas 
abarcativas, pero siempre insuficientes y abiertas a nuevos progresos, lo que constituye un gran atractivo de este campo de estudio claramente multidisciplinar. Pero comencemos por un ejemplo sencillo.

\section{La decisión en un juego de azar}

Supongamos una persona ante un sencillísimo juego de azar que da lugar a la siguiente situación de decisión: Tiene dos decisiones posibles d1, d2 representadas en el árbol de decisión ${ }^{1}$.
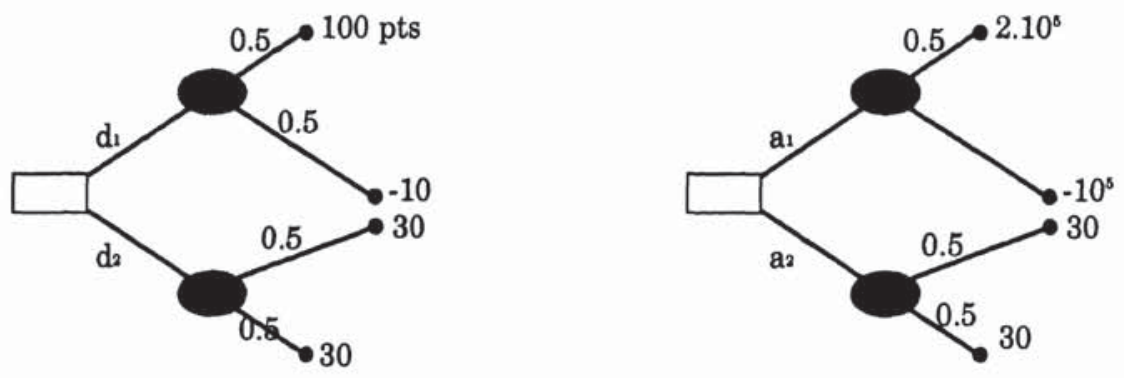

Si toma la decisión d1 el azar le da 100 ptas. Con probabilidad 0,5 y le da -10 con probabilidad 0,5 . Análogamente si se toma la decisión d2 ... . Comparar estas dos decisiones se hacía a partir de Huygens (1675), con el criterio del Máximo valor monetario esperado (que representa la ganancia media en una serie ilimitada de partidas)

$$
\begin{aligned}
& \mathrm{E}\left[\mathrm{d}_{1}\right]=0,5 \bullet 100+0,5(-10)=45 \\
& E\left[\mathrm{~d}_{2}\right]=0,5 \bullet 30+0,5 \bullet 30=30 \\
& \mathrm{E}\left[\mathrm{d}_{1}\right]>\mathrm{E}\left[\mathrm{d}_{2}\right] \text { luego } \mathrm{d}_{1}>\mathrm{d}_{2}(\text { preferido })
\end{aligned}
$$

Pronto surgió una grave objeción con el Ejemplo de Bernoulli (1738) (árbol de decisión) ${ }^{2}$

$$
\begin{aligned}
& \mathrm{E}\left[\mathrm{a}_{1}\right]=0,5 \cdot 2 \cdot 10^{5}+0,5 .-10^{5}=0,5 \cdot 10^{5} \\
& \mathrm{E}\left[\mathrm{a}_{2}\right]=0,5 \bullet 30 \times 0,5 \bullet 30=30
\end{aligned}
$$

$\mathrm{E}\left[\mathrm{a}_{1}\right]>\mathrm{E}\left[\mathrm{a}_{2}\right]$ y la solución natural para un hombre rico es $\mathrm{a}_{1} \mathrm{a}_{2}$, mientras para un pobre debe ser $a_{1}<a_{2}$, pues con a1 puede arruinarle al perder.

Surge la Regla de Bernoulli: Máximo valor moral esperado, que toma como criterio el valor esperado del logaritmo del valor monetario. 


\section{Racionalidad y toma de decisiones hacia el siglo XXI}

Bernoulli planteó, en este y otros ejemplos clásicos, con extraordinaria claridad el problema; pero su solución es bastante criticable. En primer lugar, después de haber insistido en el aspecto subjetivo del problema de la utilidad empieza por dar una función de utilidad o preferencia en certidumbre, sin tener en cuenta que se trata de preferencias en incertidumbre. En segundo lugar sigue aceptando la idea de la esperanza matemática, que había resultado de la aplicación a problemas de pruebas repetidas, olvidando que aquí se trata en general de un problema en que un individuo participa en el juego una sola vez.

Han de pasar doscientos años hasta que Von Neumann y Morgenstern (1943) dan una solución satisfactoria a este problema fundamental de la decisión en incertidumbre. Las dos características que parecen necesarias para la aparición del nuevo paradigma de la utilidad esperada, a saber, la progresiva axiomatización de la matemática (Hilbert, Kolmogoroff,...) y las fuertes necesidades de las aplicaciones económicas militares,... consecuencia de la segunda Guerra Mundial, se dan cuando Von Neumann y Morgenstern se interesan por tal problema, y consiguen su solución. Solución que no habían logrado ni Laplace ni Gauss, a pesar de que a comienzos del siglo XIX estaban en posesión de esquemas y recursos fundamentales como la probabilidad subjetiva, la fórmula de Bayes, la tabla de decisión de Pascal, la función de perdida,...

Cualidades fundamentales del nuevo enfoque sobre la decisión son: $1^{\circ}$ el concepto de preferencia se extiende no solo al dinero, sino a objetos complejos (automóviles, viajes en avión, enfermedades, días de curación,...); $2^{\circ}$ la utilidad se asocia al concepto de probabilidad mediante la idea de comparar situaciones simples aleatorias y deterministas para pasar a situaciones complejas mediante axiomas de racionalidad sencillos que conducen a la regla de oro de la máxima utilidad esperada (M.U.E.), fundamental para el Análisis de decisiones, Teoría de juegos, Negociaciones, Reconocimiento de patrones, Inteligencia artificial,...

Inspirados en la medida de las magnitudes de la Física y en sus observaciones psicológicas de las partidas de póker de Princeton, introducen VN-M las ideas radicalmente nuevas de relación de preferencia sobre las loterías y la operación natural de mixtura. Con esto establecen como modelo teórico una estructura de preorden completo sobre un conjunto de loterías, que verifican la operación lineal de mixtura, un axioma de substitución y un axioma de continuidad. Con ello demuestran la existencia de una esperanza de utilidad, que es un funcional lineal isótono, fiel y único, salvo una transformación lineal.

En definitiva, podemos decir con palabras más corrientes, que en la aplicación practica, esto se traduce en que si las preferencias de un decisor obedecen unos ciertos axiomas de gran fuerza intuitiva, este decisor debe 


\section{Sixto Ríos}

comportarse como si tratara de maximizar la utilidad esperada (principio de máxima utilidad esperada M.U.E.). Las dos diferencias fundamentales que hacen que la utilidad de Von Neumann resuelva el problema de Bernoulli son: $1^{\circ}$ que refleja las preferencias en riesgo del decisor en cualquier situación concreta por complicada que sea como consecuencia de sus preferencias subjetivas en situaciones simples y $2^{\circ}$ al considerar una situación única de decisión no utiliza para nada la repetición de juegos en las mismas condiciones y la ley de los grandes números que permite pasar de la ordenación de unas cuantas loterías sencillas de un cierto conjunto a la ordenación de otras dos cualesquiera del mismo conjunto mediante unos razonamientos y cálculos sencillos que pueden considerarse como una prolongación probabilística natural de la lógica deductiva ordinaria de Aristóteles-Boole al campo de la valoración de perspectivas aleatorias.

Como se ve la modelización matemática en el campo de la teoría de la decisión tiene un carácter distinto del que tiene en física, ya que no se trata de reconocer y reproducir mediante relaciones matemáticas un fenómeno físico, como p.e. el movimiento de un péndulo, sino de crear un modelo cuya aplicación conduzca a las decisiones que consideramos que "deben ser las mas adecuadas" a la situación con que nos enfrentamos. Es decir, no nos proponemos llegar a un modelo descriptivo sino a un modelo normativo. En palabras de Savage: establecer un conjunto de axiomas que implican una afinada percepción de los valores e incertidumbres que satisfacen ciertas condiciones de coherencia que adoptamos en nuestras decisiones.

Esta idea, que aparece claramente por primera vez en Von Neumann, ha sido posteriormente aplicada a conseguir modelos normativos para decisiones colectivas, polietápicas, dinámicas, juegos, negociaciones, etc.

Con el camino iniciado por Bayes con su fórmula famosa, preparado por Wald considerando al estadístico como un decisor, y de Finetti, con su enfoque práctico de la probabilidad subjetiva y, sobre todo, por el genial trabajo fundamental de Von Neumann (1944), logra Savage (1954) una construcción axiomática conjunta de la probabilidad subjetiva y la utilidad que generaliza el principio de M.U.E. para pasar a un principio en que las probabilidades se determinan subjetivamente por el decisor, máxima utilidad subjetiva esperada (M.U.S.E.), punto de partida de la metodología bayesiana de la decisión, hoy muy generalmente aceptada como una importante rama de la Ciencia de la decisión.

\section{Validación de modelos de decisión}

Un aspecto fundamental de la modelización de un proceso empírico es la validación del modelo que nos llevará a aceptarlo para su uso posterior o a rechazarlo para reiniciar el proceso de modelización. 


\section{Racionalidad y toma de decisiones hacia el siglo XXI}

Se dice que un modelo de un cierto proceso o fenómeno real es válido si su comportamiento representa adecuadamente el comportamiento del sistema real bajo todas las condiciones de interés.

$\mathrm{Si}$ podemos establecer un experimento en que las respuestas del modelo discrepan de algún modo de las del sistema real, dentro del área de interés para la que ha sido establecido el modelo, diremos que éste no es valido.

Como hemos indicado la demostración del principio de máxima utilidad subjetiva esperada, reduce la elección entre dos loterías complejas a la elección entre algunas otras realmente simples, y esta reducción se logra aplicando únicamente los axiomas básicos de racionalidad admitidos por el decisor. Pero al considerar la toma de decisiones por individuos desconocedores de este principio se ha observado que el proceso ingenuo "de toma de decisiones" presenta desviaciones estereotípicas de los axiomas lo que, por otra parte, es natural aun en problemas sencillos.

Creemos que más bien lo que sería extraño es que los individuos corrientes o, incluso iniciados, fueran capaces, en algunos minutos, de suplir la sabiduría acumulada de Bernoulli a Von Neumann, en este tema. Esto está en la misma línea que los frecuentes errores entre algunos profesionales (médicos, economistas, etc.) al estimar o interpretar probabilidades o manejar razonamientos probabilísticos inductivos o suplir intuitivamente el teorema de Bayes. Ello confirma la necesidad de enseñar las cuestiones fundamentales del Cálculo de Probabilidades y del Análisis de Decisiones a las personas implicadas en la toma de decisiones y, al mismo tiempo, explica la excesiva fuerza probatoria que atribuimos a los ejemplos de Allais que, en los años 50 sembraron cierta desconfianza en la validez de estos axiomas como representación del comportamiento humano natural, es decir, en que la hipótesis de la utilidad subjetiva esperada se pueda considerar la base de una teoría descriptiva de la decisión.

Durante los 70 un gran número de trabajos y experimentos de falsación, siguiendo las líneas de los psicólogos Kahneman-Tversky, significaron la tendencia a construir una teoría de la decisión descriptiva de los pasos dados por distintos individuos al enfocar y tratar de resolver "problemas de decisión de laboratorio".

Este tipo de trabajos críticos, que incluso se extendieron a la lógica aristotélica, con los experimentos de Wason, estimularon en los 80 el nacimiento de un cierto número de metodologías que explicaban unos u otros defectos que algunos habían encontrado en la teoría de la utilidad subjetiva esperada.

Desde entonces dos son las direcciones dominantes: a) construir una teoría descriptiva pura, a partir de datos de experimentos y de las intuiciones resultantes del estudio de los mismos, como es el caso de la "teoría 


\section{Sixto Ríos}

de prospectivas" de Kahneman-Tversky; o bien, b) debilitar o modificar algunos de los axiomas de Von Neumann-Savage, para llegar a una más amplia aceptabilidad como ha hecho, por ejemplo, Fishburn, con su notable teoría de la utilidad bilineal simétrica, y otros muchos en diferentes direcciones. Pero como dice el propio Fishburn (1988), las nuevas teorías que tratan de ajustarse mas a las observaciones de decisores reales no refuerzan la sencillez y elegancia del modelo M.U.S.E.

Sin embargo, estos trabajos experimentales continúan, como se ve a través de las actas de los congresos FUR, especialmente dedicados a la teoría de la utilidad, en un proceso dirigido al logro de un modelo sencillo, coherente, bello y, por supuesto, racional que sea a la vez aceptablemente descriptivo del comportamiento natural de los decisores mas o menos ingenuos.

Se trata de un gran programa de investigación en la nomenclatura de Lakatos, al que hemos dedicado una parte de nuestro trabajo y de algunos de nuestros discípulos en diversas etapas de nuestra actividad científica. Lakatos (1970) propone, en efecto, que una teoría científica como la de la utilidad esperada, se considere como un programa de investigación científica constituido por un núcleo duro formado por una hipótesis mantenida, no susceptible de refutación dentro del marco de trabajo, y un cinturón protector de hipótesis contrastables que se van ajustando a la luz de la nueva evidencia empírica. Los programas de investigación contienen, en general, una heurística negativa que excluye hipótesis, no consistentes con el núcleo y una heurística positiva que sugiere el tipo de trabajo que se debe hacer para engendrar y contrastar hipótesis refutables.

Precisamente en esta línea se encuentran los trabajos que hemos realizado en la Real Academia de Ciencias de Madrid, en colaboración con el Grupo de Análisis de Decisiones del Departamento de Inteligencia Artificial de la Universidad Politécnica de Madrid, de la Universidad Rey Juan Carlos, el Instituto de Medicina Experimental del hospital Gregorio Marañón (Madrid), la Secretaría General Técnica del Ministerio de Defensa, el Departamento de la Economía de la Universidad Carlos III y el Departamento de Estadística de la Universidad de Málaga, y así mismo las colaboraciones de los Departamentos de Estadística de la Universidad Complutense, UNED, Tetuan,...

Este trabajo, desarrollado con el patrocinio de la fundación del Banco de Bilbao-Vizcaya, durante dos años académicos (93-94), ha permitido contrastar y mejorar la llamada teoría robusta de la decisión propuesta en su tesis por David Ríos, que suprime la condición de completitud en el orden y debilita el axioma de continuidad, lográndose una metodología que, complementada con el estudio profundo de la sensibilidad, mejoró satisfactoriamente tratamientos anteriores. 


\section{Racionalidad y toma de decisiones hacia el siglo XXI}

En resumen, diremos que en este tema -nunca agotado- de la validación de la Teoría de la Utilidad, no debemos olvidar que, hoy por hoy, no se puede considerar ninguna teoría como una dogma intocable para la eternidad y que continuara siendo útil y necesario el aprendizaje a través de los estudios empíricos y de los experimentos realizados con personas o sistemas decisionales: empresas, comités, tribunales, ..., que toman decisiones y que han permitido, y continuaran permitiendo, perfeccionar las teorías de la decisión.

Si nos situamos a un nivel de la práctica corriente, podemos decir que una conducta usual es aceptar que la validación de un método formalmente correcto, empieza en que sea localmente correcto, es decir, aceptable para problemas menores (situaciones sencillas en que la solución técnica coincide con la intuitiva). Después se amplia el dominio de problemas tanto en complejidad como en tamaño, considerándose que se ha conseguido un método de confianza superior al del intuitivo directo, aunque formalmente no tenga sentido tratar de "demostrar" tal superioridad. Mas bien se trata de ir convenciéndose de que si se incorporan estas metodologías racionales, compartidas cada vez mas por analistas de decisiones e investigadores de distintos campos, se va logrando un incremento significativo de progreso científico superior al que se lograría si cada uno actuara sin tenerlo en cuenta. Tal es la idea básica de la teoría prescriptiva de la decisión, como síntesis de teorías normativas y descriptivas, punto de partida del fecundo Análisis de Decisiones actual.

\section{Análisis de decisiones}

Hemos dado unas breves indicaciones de los fundamentos de la teoría de la decisión, que es la base del Análisis de Decisiones, cuyo germen se encuentra ya en el libro de Von Neumann-Morgenstern, al introducir las dos formas clásicas de representación de juegos, a saber, tablas y arboles de decisión. Las tablas o matrices de decisión, cuyo antecedente más remoto es la tabla de Pascal, permiten una representación natural y directa en muchos problemas en que aparecen en forma explícita alternativas, estados y consecuencias; pero es más frecuente que se busque y obtenga una representación detallada explícita de todos los escenarios que pueden aparecer en el curso de un proceso de decisión, es decir, lo que se llama un árbol de decisión.

La idea directriz en este enfoque es la consideración profunda de los elementos y aspectos, no necesariamente numéricos, de los problemas, comenzando por lo que podríamos llamar modelización estructural, porque incluso puede ser más fácilmente comprendida por los decisores o 


\section{Sixto Ríos}

clientes (médicos, administradores,...)que los aspectos de asignación y cálculo numérico efectivo, que tradicionalmente constituyen el dominio de los especialistas por implicar en su operacionalismo la metodología probabilística bayesiana u otras. Estos aspectos cuantitativos se incorporarían al modelo en una segunda etapa, que será mejor aceptada una vez que el decisor ha penetrado en el esquema presentado por el analista de decisiones. En definitiva, tal modelo deberá ser una consecuencia del intercambio y ajuste entre el modo intuitivo de pensar del decisor y la ayuda científica del analista. Este será el camino para llegar al que se suele llamar modelo requisito, satisfactorio para el analista o teórico de la decisión y para el decisor o práctico de la decisión.

De los dos aspectos fundamentales que se manejan para llegar a la selección de alternativas de decisión, a saber, las preferencias y creencias, puede decirse que las primeras son más fáciles de comprender por el decisor, gracias a los axiomas sencillos e intuitivos que les atribuimos para su manejo. En cambio, es bastante reciente, gracias a trabajos de Dawid, Smith, Pearl, Allard, ..., el haber concretado el concepto de relevancia, como fundamental para la relación de inferencia entre variables inciertas, gobernado por los axiomas de Dawid.

Basado en estas directrices, el enfoque tradicional del diseño de un árbol de decisión se reduce a construir paso a paso un grafo de tipo árbol, en que el origen representa la situación inicial y el resto del árbol representa, en orden cronológico, los movimientos que corresponden al decisor y los que tienen carácter de azar o incertidumbre. De los primeros parten arcos o ramas que corresponden a alternativas para el decisor y de los segundos los que representan sucesos posibles.

Tras la laboriosa asignación de utilidades y probabilidades condicionadas, se llega finalmente al modelo del problema ya en forma numérica. La propiedad fundamental de la utilidad esperada, asociada al método regresivo de la programación dinámica permite, partiendo de los nodos últimos en que se encuentran las utilidades finales, regresar, obteniendo en cada paso el nodo de decisión al que corresponde máxima utilidad esperada, para llegar, finalmente, al origen con la solución deseada.

En todos estos campos se acepta hoy como necesario el uso de los métodos del análisis de riesgos y decisiones que en el ingreso cognitivo debe hacerse a través de la medida de la incertidumbre y de la preferencia mediante probabilidades subjetivas y utilidades.

En definitiva, los métodos que se emplean consisten en utilizar las opiniones de expertos en el campo especial considerado como una primera aportación para la asignación de probabilidades subjetivas. Tal aportación ha de someterse a algunas operaciones de control como la calibración, y otras que permiten, análogamente a como se hace en otros 


\section{Racionalidad y toma de decisiones hacia el siglo XXI}

campos de medidas más clásicas, aproximarse, paso a paso, a una medida más fiable.

La aplicación del método de la esperanza de utilidad a situaciones concretas requiere, además de la determinación de las probabilidades de los sucesos que pueden presentarse, la de la curva o función de utilidad del decisor, cuyo punto de apoyo teórico es el axioma de continuidad. En el caso de las decisiones con multicriterios, el llegar a la construcción efectiva de la función de utilidad del decisor es un problema dificil que se ha conseguido realizar, en la práctica, gracias a la introducción de nuevos conceptos como la independencia preferencial, independencia en utilidad,..., en los que se basa la construcción efectiva de los llamados sistemas de soporte a la decisión, que permiten la resolución de problemas muy complejos del Análisis de Decisiones.

\section{La racionalidad limitada de Simon y otros puntos de vista}

El premio Nobel Prof. Simon, en un artículo reciente escrito conjuntamente con Dantzig, Raiffa,..., ha dicho que "el desarrollo de la teoría de la utilidad subjetiva esperada es una de las grandes conquistas intelectuales del siglo XX, que nos da por primera vez un principio formalmente axiomatizado que permite a un individuo comportarse de una manera consistente y racional".

"Admitiendo probabilidades asignadas subjetivamente, la teoría de la utilidad esperada subjetiva abre el camino para fusionar opiniones subjetivas con datos objetivos, un enfoque que puede ser utilizado también en sistemas decisionales con hombres y máquinas. En la versión probabilística de la teoría, la regla de Bayes prescribe como los individuos deberían tener en cuenta la nueva información y como deberían responder a la información incompleta".

Se ve, pues, que Simon, uno de los más agudos críticos de la teoría de la utilidad esperada durante 40 años, acepta su enorme importancia para la obtención práctica de las decisiones en incertidumbre en universos bien definidos y de limitada complejidad. Pero también considera que hay situaciones reales de decisión a las que no todos los axiomas de la teoría de la utilidad subjetiva esperada se ajustan de una manera empíricamente aceptable. Propone entonces las teorías que llama de racionalidad limitada, que cambian algunas de las hipótesis de la teoría racional clásica.

Por ejemplo, en vez de suponer bien definido un conjunto fijado de alternativas entre las que el decisor ha de elegir, admite un proceso para la génesis de alternativas posibles. En vez de suponer distribuciones de probabilidad conocidas de los resultados, introduce solamen- 


\section{Sixto Ríos}

450

te métodos de estimación para las mismas, o bien, considera estrategias que permitan tratar la incertidumbre sin suponer conocidas las probabilidades. En vez de maximizar la esperanza de utilidad busca una estrategia satisfaciente, asociada a unas metas fijadas por el decisor. Estos cambios son sugeridos, según Simon, por el conocimiento empírico del comportamiento humano en los procesos de decisión y de las limitaciones de nuestra capacidad cognitiva para descubrir alternativas, calcular sus consecuencias, y hacer comparaciones entre las mismas.

No se trata aquí de hacer una comparación de las ideas directrices de esta y otras teorías, llamadas de racionalidad limitada, que ruedan hace más de 40 años y pretenden inspirarse más en el estudio sistemático y detallado del desarrollo del proceso humano de toma de decisiones, que en el planteamiento de la situación predictiva de elección en la forma que lo hace la teoría de la utilidad esperada.

Trabajos recientes (T.K. Lant, 1994) han iniciado la comparación experimental de estos dos grandes tipos de modelos, apareciendo una tendencia a considerarlos compatibles y, en cierto modo, complementarios.

$\mathrm{Y}$ otro tanto podríamos decir sobre algunas teorías basadas en las jerarquías analíticas (Saaty), trayectorias (Wierbizki), conjuntos difusos (Zadeh), riesgo fijado (Ríos, Girón, Layachi,...) siempre que tengan las características esenciales de ser observables, medibles y coherentemente modelizables.

\section{Aplicaciones del análisis de decisiones: hacia el siglo XXI}

Un progreso reciente surgido precisamente de los éxitos del Análisis de Decisiones en los problemas de decisiones individuales monocriterio, es la aplicación efectiva de este Análisis a problemas mucho mas complejos llamados de multicriterios, dinámicos, multiniveles, polietápicos, decisiones sociales, colectivas, juegos, negociaciones,...

Muchos de estos procesos de decisión, estudiados en sus comienzos, con lo que podríamos llamar modelos magníficos, creados por sus fundadores y correspondientes escuelas, encontraron un ritmo de desarrollo mas lento en etapas posteriores, en que se empezó a echar de menos la posibilidad de tratar de una manera más real los problemas que se presentaban en la realidad.

Como dice Raiffa en su importante libro sobre Negociaciones: "Existen bellas teorías económicas de la empresa que explican, en una primera aproximación, como las empresas se comportan y como deberían comportarse. Pero cuando uno se sitúa próximo a los problemas actuales de 


\section{Racionalidad y toma de decisiones hacia el siglo XXI}

los directores de empresas, estas teorías generales resultan demasiado vagas para ser relevantes. Al nivel de la empresa, lo que es necesario, entre otras cosas, es un conjunto de herramientas analíticas mediante las que un equipo de especialistas pueda interactuar sobre una base consultiva con los decisores empresariales. Estoy hablando no solo de los investigadores operativos y analistas de decisiones, sino también de especialistas en finanzas, mercados y otras áreas funcionales de la empresa".

Pues bien, el Análisis de Decisiones, con sus modernas versiones y la profundización en la Teoría de la Utilidad, ha sido capaz de adaptarse apropiadamente y resolver tales problemas llegando a soluciones y recomendaciones concretas.

He aquí brevisimamente algunas referencias:

a) Decisiones multicriterio: importantes problemas de decisión multicriterio en ambiente de incertidumbre con una jerarquía en los criterios, en varias o infinitas etapas, con varios grupos de personas afectadas de distinta forma y varias personas participando en la decisión, etc., se presentan constantemente en la realidad político-social y es inevitable estudiarlos y resolverlos con herramientas mas precisas e incisivas que la intuición de los expertos.

a, 1) Una cuestión importante es la comparación de las consecuencias de diferentes maneras posibles de suministrar en el futuro la energía necesaria a una región de millones de individuos, mediante las diferentes fuentes posibles como carbón, petróleo, gas, viento, energía nuclear, hidroeléctrica, solar. Según las proporciones relativas que adoptemos, tendremos alternativas con muy distintos impactos desde los puntos de vista de calidad ambiental, sanidad, seguridad, situación socioeconómica de los seres que habitan las distintas regiones y sobre el sistema de costes, y el problema es buscar la combinación optima o, al menos, una satisfaciente. Y una idea de la magnitud e importancia del problema se tiene al considerar que las decisiones tendrán consecuencias para muchos años y muchos individuos.

a, 2) Selección de flotas de aviones y, en general, de ofertas en las compras de armamento, teniendo en cuenta múltiples criterios, concurrencia, incertidumbre,...

a, 3) A nivel individual, los métodos de Análisis de decisiones constituyen el enfoque adecuado a los problemas de formación de una cartera de valores (Portfolio Selection) en que son fundamentales los trabajos de los Premios Nobel Markowitz, Black, Scholes, ..., asimismo la compra de un coche, de una casa o, incluso, de los alimentos para el consumo diario.

a 4) La inclusión de objetivos múltiples en los procesos de planificación, superando a los procesos clásicos con un solo criterio económico es 


\section{Sixto Ríos}

impuesto o recomendado ya por las leyes en algunos países como método obligatorio al tratar del aprovechamiento de recursos naturales como el agua, las minas, etc. (ver, por ejemplo, USA Water Resources Council, 1970).

a,5) en el ámbito internacional hay que señalar que la UNIDO (United Nations Industrial Development Organization) ha publicado ya en 1972 unas normas para evaluación de proyectos que los países en desarrollo deben tener en cuenta, objetivos múltiples: empleo, redistribución de la renta, balanza de pagos, consumo agregado,...

\section{b) La racionalidad colectiva y las votaciones}

Como pasar racionalmente de las decisiones individuales, consecuencia de los juicios opiniones y percepción de la incertidumbre de un conjunto de personas (tribunal, comité, jurado, consejo de administración,...) a una decisión única de grupo.

b, 1) Amalgamación de ordenaciones. La opinión generalizada entre los economistas de la carencia de sentido de la amalgamación de utilidades individuales llevó al Prof. Arrow a estudiar el problema de amalgamación de preferencias individuales para obtener una función de preferencia social en su libro famoso de 1951.

El punto de partida de estos estudios está en los trabajos sobre las votaciones debidos a Borda, Laplace, Dogson y, sobre todo, en la famosa paradoja de Condorcet (1785) en que se ve como falla la transitividad en las decisiones colectivas, tomadas con la regla democrática de la mayoría.

Arrow se plantea la cuestión de que criterios mínimos deben satisfacer las preferencias sociales obtenidas a partir de las preferencias de un conjunto de individuos que forman tal sociedad.

"Si excluimos la posibilidad de comparaciones interpersonales de la utilidad -dice Arrow- los únicos métodos satisfactorios de pasar de las preferencias individuales a las sociales para conjuntos amplios de individuos son las impuestas o las dictatoriales".

Este decepcionante resultado de las primeras investigaciones de Arrow, desanimó, al principio, a muchos investigadores de proseguir el estudio de las decisiones colectivas. Pero pronto las cosas cambiaron y como muchos otros teoremas negativos famosos (como el teorema de Gödel, o el teorema de Banach-Tarski) dio lugar a una extensísima serie de trabajos que hoy continua, tratando de aclarar el alcance de sus condiciones y las consecuencias de las modificaciones de las mismas. 


\section{Racionalidad y toma de decisiones hacia el siglo XXI}

Trabajos posteriores han logrado primero la rehabilitación de la regla de la mayoría de un modo completamente general para dos opciones y, de un modo muy amplio, para n opciones. Es decir, las conocidas objeciones a la regla de la mayoría por no transitividad (paradoja de Condorcet), etc., desaparecen en tales condiciones similares a muchas situaciones reales: votaciones con dos partidos políticos o con un conjunto de partidos que admitan una cierta ordenabilidad de sus opiniones.

Hemos traído a recuerdo todos estos problemas de gran interés práctico y teórico, porque también en ellos tienen aplicación eficaz los conceptos del análisis de decisiones. Por ejemplo, trabajos recientes de d'Alessandro (1994), relativos al proceso de amalgamación de decisiones multicriterio de un conjunto de $\mathrm{p}$ decisores, entre $\mathrm{m}$ alternativas, cuyos resultados o medidas con $n$ criterios dan lugar a espacios cuyos elementos son matrices $\mathrm{m} \times \mathrm{n}$ y el nuevo concepto de conjunto eficiente da lugar a resultados que pueden conducir a defensa ciega de intereses personales, riesgo de parálisis de negociaciones, dilución de responsabilidad... Esto lleva a la necesidad de modificar la estructura del problema de modo que se reconduzca a una fuerte comunidad de espíritu, y una autoridad superior que represente intereses generales.

\section{b, 2) Decisiones políticas y econometría}

En la línea de los trabajos sobre decisiones colectivas están las aportaciones del Premio Nobel de Economía Prof. Ragnar Frisch, que ya en su discurso en la Academia Sueca (1971), llama a la cooperación entre políticos y econometras para que formalicen la función de preferencia, que debe considerarse como la base del concepto de política óptima.

Considera la función de preferencias simplemente como una función de algunas de las variables, que permiten describir una economía (regional, nacional, mundial), de modo que la maximización de tal función sea equivalente a la definición del objetivo que quiere lograr la política económica. No se trata, pues, de la función de bienestar, tratada en forma teórica en economía.

"Tengo la firme convicción -dice Frisch- de que en una aproximación a la política económica por la vía de la función de preferencia está la clave de una reforma de los métodos de decisión de las sociedades, absolutamente necesarios en el mundo actual".

"Hay dos aspectos en la metodología: a) el establecimiento de los objetivos a través de la función de preferencia, y b) la construcción de un modelo con ecuaciones y condiciones, bajo el cual caminemos hacia los objetivos". 


\section{Sixto Ríos}

"La función de preferencia es la que aplica la autoridad existente a las decisiones que deben tomarse n la sociedad. La autoridad puede ser un comité o un parlamento. En el ütimo caso la función de preferencia es la amalgamación a través de la maquinta política de las funciones de preferencia de los diversos partidos políticos". El sentido y realismo de su consideración y construcción aparece clara para Frisch, tras sus contactos y trabajos con varios jefes de gobierno y ministros de economía de distintos países.

"Mediante métodos de interviú sofisticados se puede formalizar en una serie de etapas sucesivas con la colaboración del político y el econometra y, por supuesto, con el auxilio del ordenador, la función de preferencia de cada partido político. Paso a paso podrá llegarse, con esta metodología, a una función de preferencia con la cual se obtenga la evolución prevista de la sociedad de la que el político diga "justo es lo que yo deseo". O bien, se hará ver al político que sus desiderata son, por ahora, inalcanzables simultáneamente y que se ha de conformar con objetivos más modestos".

"De este modo se puede lograr que cada partido político reconozca las consecuencias de su actitud y admita estas consecuencias públicamente. Con esto se habrá ganado bastante claridad en las discusiones entre partidos".

"Pero aún se puede ir más lejos, se puede tratar de lograr un compromiso político sobre la formulación de una función de preferencia numérica unificada".

"En consecuencia, en un país democrático, el Parlamento, suprema autoridad política, deberá emplear la mayor parte de su tiempo y energía sobre la discusión de esta forma de compromiso y sobre las consecuencias que tal forma implicaría; en vez de utilizar prácticamente todo su tiempo y esfuerzo en decidir sobre medidas individuales que pueden haber sido propuestas. Este último podrá llamarse método prehistórico". Creo que valía la pena esta larga cita de una autoridad científica como Frisch, porque pone de manifiesto la posibilidad de llegar a resultados concretos aceptables en importantes problemas de gobierno en un país.

\section{b, 3) Conferencias de decisión o brainstorming}

En 1986 un grave accidente nuclear tuvo lugar en Chernobyl (URSS), quedando completamente destruido un reactor, que estuvo vertiendo sustancias radioactivas durante 10 días, que contaminaron grandes extensiones de tierra y pueblos. Cuatro años después fue requerida por las autoridades soviéticas la formación de una comisión coordinada por la IAEA, para que estudiara los diversos efectos de la catástrofe sobre la población y la influencia de las medidas iniciales tomadas por la URSS para aliviar los efectos sobre las poblaciones. Inicialmente se había pensado la no conveniencia de tener en cuenta solamente los aspectos de protección radioló- 


\section{Racionalidad y toma de decisiones hacia el siglo XXI}

gica, sino considerar también otros factores de tipo sociológico, político,... De modo que surgió la necesidad de aplicar la técnica de la decisión multiatributo para investigar el difícil y apasionante problema.

La catástrofe de Chernobyl, puso de manifiesto la necesidad de un plan para aliviar con urgencia en el futuro las consecuencias de este tipo de graves accidentes. RODOS (Real time on line decision support) es el nombre con que se bautizó tal sistema, a cuyo establecimiento se llegó tras tres "conferencias de decisión” dirigidas por el estadístico y decisiólogo Prof. French de la universidad de Manchester. Este sistema de T.D. mediante un grupo de experimentos en A. D. y especialistas en el problema técnico, dirigidos por un analista de decisiones, tiene como objetivo llegar a una solución aceptada por el grupo en un problema previamente estudiado individualmente. No suele haber votaciones en estas conferencias y el informe final es un conjunto de conclusiones y de acciones que se deben tomar para implantar la decisión final que conduzca a los resultados esperados.

\section{b, 4) Incertidumbre endógena, cambios climáticos y ecología}

Actualmente el estudio de los riesgos producidos por los discutidos cambios climáticos tiene dos novedades fundamentales para su estudio: a)su carácter global, es decir, que afectan a todas y cada una de las regiones del planeta, y b) su carácter endógeno, es decir, son causados por la misma actividad económica (por ejemplo, las emisiones industriales de $\mathrm{CO}_{2}$ y CFC). Ambas características contribuyen a que la tradicional incertidumbre se complique cualitativa y cuantitativamente en los problemas de predicción climática.

El carácter endógeno de la incertidumbre en estos problemas permite tomar medidas de mitigación, mediante decisiones para reducir las causas de los cambios climáticos. Contrariamente, los seguros no pueden hacer nada para reducir la probabilidad de daños (riadas, avenidas, desertización, extinción de especies, enfermedades,...) debidas a los cambios climáticos. Solo pueden establecer compensaciones para los afectados por tales desastres. De esto se derivan los problemas, de si es posible y como adaptar los seguros a este nuevo tipo de riesgos difícilmente estimables, endógenos, colectivos e irreversibles y también cuales son las limitaciones o modificaciones a imponer a la tecnología productiva. Trabajos recientes de Arrow-Debreu, Chichilnisky, Heal, etc. , han iniciado algunas soluciones a estos problemas de decisión, en que están muy interesados organismos internacionales como las Naciones Unidas, Banco Mundial, Comunidad Europea,...

\section{b,5) Decisiones polietápicas}

Un refrán bien conocido: "Mas vale pájaro en mano que ciento volando" refleja muy bien el sentido del cambio del valor de un objeto o canti- 


\section{Sixto Ríos}

dad monetaria en el paso del tiempo. Dentro de este orden de ideas resulta especialmente interesante considerar las decisiones de inversión, en que al adquirir un bien se espera una sucesión de beneficios en tiempos posteriores. En definitiva, la comparación de inversiones se reduce a la comparación de complejos de $n$ componentes o cantidades que se espera representen los resultados de la inversión en el año cero y sucesivos.

Una axiomática, debida al Premio Nobel Prof. Koopmann, permite reducir cada complejo a un número real, que es su valor actualizado, a través de la conocida tasa de descuento y con esto se resuelven, de manera simplista, este tipo de problemas de inversiones, como si fueran de multicriterios en certidumbre.

Su papel es importante para platear correctamente los problemas de decisión, dinámicos o secuenciales o de control estocástico, juegos diferenciales, como el problema de regulación de una presa, etc.

En época reciente se ha estudiado la analogía profunda entre la incertidumbre y el factor tiempo. Por ejemplo, muchas personas prefieren el resultado de una lotería como $(0.95 ; 1000 ; 0.05 ; 0)$ en este momento ha 1000 ptas. seguras dentro de un año. Mazur realizo en 1987 una serie de experimentos con la alimentación de palomas que prueban que las funciones de descuento por retraso son hiperbólicas, lo mismo que las que expresan los equivalente en certidumbre probabilísticos. Estas relaciones son objetivos de investigaciones recientes para profundizar la similaridad entre selección intertemporal, selección bajo incertidumbre y ver si se pueden extender a otros tipos de selección multiatributo, tanto en animales como en personas.

\section{b, 6) Procesos dinámicos de decisión}

Un problema importante polietápico dinámico multicriterio es el de la regulación de una presa. Su propósito es, en esquema, guardar agua durante los periodos de abundancia para utilizarla en temporadas de escasez. Los objetivos que se proponen al construir una presa pueden ser, entre otros: suministros de agua a poblaciones, industrias, tierras, generación de energía eléctrica, protección contra avenidas, aspectos ecológicos y recreativos,... El problema de la regulación de servicio de un embalse, optimizando globalmente estos objetivos, presenta una gran dificultad al observar que son, en buena parte, conflictivos. A esta dificultad se añade la gran incertidumbre del proceso natural de acumulación de ingresos y de la demanda de agua para diferentes usos. Ello ha originado una serie de metodologías como diagramas de Klemens, programación lineal y no lineal, programación dinámica, modelos estocásticos,... Recientes trabajos de D. Ríos y A. Salewicz han introducido la metodología del Análisis de Decisiones bayesiano para resolver el problema de la 


\section{Racionalidad y toma de decisiones hacia el siglo XXI}

regulación del sistema del lago Kariba y otros de la cuenca del Zambeze, del que se benefician ocho países africanos. Tal trabajo, realizado (1992) bajo los auspicios del IIASA, se considera que supera las metodologías predecesoras y conduce a buenos resultados.

\section{b, 7) Decisiones interactivas: Juegos, negociaciones, asignación} de costos.

Es bien sabido que la teoría de juegos es una de las creaciones más importantes de John V. Neumann, que la propuso inicialmente para modelizar las situaciones de decisión interactivas con participación de varios decisores y consecuencias para todos ellos, inspirándose en intuiciones de los juegos de sociedad, especialmente del juego del póker.

Esta teoría atrae fuertemente el interés de matemáticos, estadísticos, economistas, psicólogos,..., en sus comienzos en los años 40 y 50, languidece después algún tiempo, para recuperar en las dos ultimas décadas todo su vigor con la aparición de profundos e importantes resultados. Pero en las posibilidades de aplicación de estos resultados se presentan dificultades, sobre todo porque la mayor parte de los conceptos tienen carácter cuantitativo: utilidad esperada, funciones de pago, medidas sobre creencias probabilísticas,...

Para hacer más viables y efectivas las aplicaciones de la teoría de juegos, se ha pensado recientemente (Allard, Smith, Raiffa,...) en construir una teoría cualitativa o estructural, que permitiera lograr resultados profundos para llegar a consecuencias realmente prácticas.

Las ideas directrices parten del importante concepto de "conocimiento común", introducido por Aumann, según el cual "cada jugador conoce lo que sus oponentes conocen y a su vez, estos conocen lo que el primero conoce que ellos conocen,..."

A partir de esta idea se admiten las siguientes hipótesis de trabajo:

El grafo del diagrama de influencia del juego es el mismo para todos los jugadores, salvo que una variable de decisión de un jugador sería una variable incierta para los oponentes.

Las deducciones hechas por los distintos jugadores mediante las reglas de irrelevancia son conocimiento común para todos los jugadores.

También es conocimiento común suponer que un jugador prefiere la decisión $d_{1}$ a la $d_{2}$, si d $d_{1}$ no es una función invertible de $d_{2}$ que da idéntica estructura de pago a $\mathrm{d}_{2}$.

Este planteamiento no requiere que todos los jugadores sigan el mismo método inferencial, bayesiano o no. Basta que sus creencias sean consistentes con el diagrama de influencia establecido para el problema, supuesto que tal diagrama sea realmente común. 


\section{Sixto Ríos}

458

Prosiguiendo este camino se esta consiguiendo una teoría cualitativa plausible, fácilmente implantable y suficientemente penetrante para hacer deducciones interesantes y profundas en situaciones prácticas.

En todo caso, este planteamiento y sus resultados se consideran de gran interés filosófico, matemático y práctico.

Esta tendencia actual a relacionar más estrechamente la teoría de juegos con el análisis de decisiones marcha paralela al desarrollo reciente de la llamada Teoría y practica de las Negociaciones dentro del programa de Raiffa (1982) y los trabajos de Neale-Bazerman (1991), Sebenius (1992),..., a la que vamos a referirnos brevemente, tras hacer una indicación sobre el problema de la:

b, 8) Asignación o reparto de costos. El problema de reparto de costos de una obra importante (presa, aeropuerto, servicios conjuntos,...) entre los distintos usuarios, presenta una estructura formal análoga a la teoría de juegos cooperativos. Por ejemplo, en el caso del aeropuerto, cada aterrizaje de un avión es una jugada y $\mathrm{v}(\mathbf{S})$ es el coste de construir y mantener el aeropuerto para realizar un conjunto $S$ de aterrizajes; pero $v(S)$ no depende solo del número de aterrizajes en $\mathrm{S}$, sino de sus características, que son diferentes, por ejemplo, en un 747 que en un Airbus. El método axiomático y el análisis de decisiones son especialmente atractivos en estos problemas a los que dan soluciones particularmente transparentes (Billera, Heith,...).

b, 9) Negociaciones. Los enfoques científicos de los problemas de negociación y mediación van precedidos de una larga tradición en las ciencias político-sociales, como se observa en la lectura del famoso libro El Príncipe (1515) de Maquiavelo. Tradicionalmente el campo natural de estudio de los procesos de negociación y mediación ha correspondido a los sociólogos, psicólogos, diplomáticos, politólogos, ... La importancia de estos estudios se ha visto reforzada en situaciones críticas recientes en que del resultado de negociaciones sobre el desarrollo de las armas nucleares ha dependido fuertemente, incluso, el futuro de la humanidad. Ello ha conducido a la formación de importantes equipos multidisciplinarios de especialistas, como en el proyecto de la Universidad de Harvard o los de la Arms Control and Dissarmament Agency de Washington, a cuya reunión de 1968 (agosto) tuve el honor de asistir como observador.

La labor de estos equipos de trabajo ha contribuido a que se vayan construyendo una serie de modelizaciones matemáticas que permiten el conocimiento profundo de los procesos de negociación, extraordinariamente complejos y variopintos. Pero, como ha dicho Raiffa: "Existen bellas teorías del proceso de negociación que explican en una primera apro- 


\section{Racionalidad y toma de decisiones hacia el siglo XXI}

ximación como se comportan o debían comportarse los negociadores. Pero lo que se necesita es el uso creativo del pensamiento analítico que explote las técnicas analíticas que ya existen".

La metodología del proceso de negociación implica una sucesión de etapas con objetivos limitados, en que cada uno de los agentes toma una decisión que le aproxima a uno de sus objetivos. Tal decisión es presentada posteriormente al oponente que, a su vez, la transforma en una nueva propuesta, etc.

$\mathrm{Al}$ tratar de establecer un método analítico para resolver estos problemas, sea entre dos partes monolíticas o entre varios colectivos, se suele partir de la consideración de cuatro elementos básicos: a) intereses de las partes, b) alternativas para llegar a un convenio, c) creación y proclamación de valores, y d) movimientos para cambiar el desarrollo del juego.

Se comienza por modelos en que participan dos adversarios negociando sobre un solo resultado, para pasar después a resultados múltiples y, mas tarde, a dos o más coaliciones con resultados múltiples, etc. Muchas nociones y recursos de la teoría de juegos cooperativos y teoría del aprendizaje se han adaptado a estas formulaciones; pero la contribución actual más importante es la de los arboles y diagramas de decisión, sistemas de soporte a la decisión, sistemas expertos, etc., que ha puesto de manifiesto en problemas prácticos importantes como las negociaciones como la ley del mar, o las de la mina "El Teniente" de Chile,..., y miles de ejemplos mas en que se van demostrando el interés del progreso en esta nueva "ingeniería de negociaciones", en que es frecuente que intervengan como adversarios representantes de países o comarcas de diferentes culturas.

b,10) Cultura y Decisión. Justamente este tipo de decisiones interactivas ha despertado el interés por la realización de estudios comparativos en relación con el comportamiento en las decisiones de individuos o grupos sociales pertenecientes a distintas culturas, no solo a nivel nacional, sino más ampliamente a nivel de grupos clasificados por etnia, edad, sexo, religión,...

El comportamiento individual y el de grupo están fuertemente influenciados por la cultura, que sabemo:s es un conjunto de características de los individuos que se refieren, fundamentalmente, a sus creencias y valores básicos, su concepto de verdad, riesgo, incertidumbre, aleatoriedad, presentan grandes diferencias de unas culturas a otras, como han observado Georgescu-Roegen y otros.

El concepto de aleatoriedad, que tan sofisticado aparece en nuestra época, en las culturas occidentales, llegando a la diferenciación con la noción de caos, dista mucho de la idea causal de "deseo de Allah", tan arrai- 


\section{Sixto Ríos}

gada en la cultura islámica, los espíritus activos en algunas culturas africanas, etc.

La componente lógica de una cultura se refiere al modo en que los individuos establecen la causacion, que va de la simple asociación de sucesos, a la evidencia de conexiones causales.

Los valores son órdenes de preferencia sobre estados de parcelas del universo, que juegan un papel importante en las consecuencias de las decisiones.

El estudio profundo de estas relaciones de ajuste entre componentes de una cultura y sistemas de organización y de decisión, ha conducido a resultados interesantes en relación con las previsiones sobre comportamientos posibles de adversarios de diferentes culturas y, en consecuencia, a obtener mejores resultados, en un problema de negociación de un tratado comercial, de un convenio internacional de pesca,...

Otro tema importante relacionado con el anterior es crear reglas, técnicas y conocimientos que sean universales, es decir, válidos para todas las culturas.

Se plantean entonces problemas como:

¿Cuáles son los métodos para descubrir la universalidad y especialidad de nuestro conocimiento?.

¿Cuáles son las realidades profundas que pueden conducir bien a reglas universales, o bien indigenizantes sólo válidas para sociedades particulares?

Hemos querido citar siquiera estos problemas apasionantes, que se estudian actualmente por equipos multidisciplinarios, cuyo interés no es solo teórico, sino aplicado, como puede verse en el estudio reciente (Eun, Resnik, 1994) del problema de diversificación de la cartera, que tiene distinto enfoque para japoneses que para americanos. En el se ha visto como estos pueden obtener, por diversificación internacional, mayores ganancias con menor riesgo.

\section{b, 11) Decisión cuántica}

La mecánica cuántica considerada generalmente como uno de los grandes descubrimientos del siglo XX, revela que gran parte del comportamiento de la materia y la radiación no puede ser descrito por la mecánica clásica ni la relativista.

A partir de los años 60 se ha iniciado una teoría de la decisión cuántica para acomodarse al estudio de los fenómenos de la Mecánica cuántica, en que no valen algunas de las suposiciones clásicas de los métodos estadísticos tradicionales.

En mecánica cuántica, el carácter aleatorio de un resultado no es una cuestión de ignorancia o falta de especificación por parte del observador. $\mathrm{El}$ origen de la aleatoriedad tiene un carácter esencial y algunos resulta- 


\section{Racionalidad y toma de decisiones hacia el siglo XXI}

dos observables violan los axiomas de Kolmogoroff de la probabilidad. Ha habido, pues, que modificar los métodos tradicionales de la decisión para llegar a una teoría de la decisión cuántica en que los conceptos centrales de la Física cuántica son mas claramente formulados y comprendidos. Así, el principio de incertidumbre de Heisenberg se puede obtener como una consecuencia de la versión cuántica de la acotación de Cramer-Rao, con la que se consigue mas información y se facilita la experimentación.

Pero no se trata sólo de aportaciones teóricas, sino que los métodos de la inferencia y la decisión estadística han proporcionado decisiones optimas y estimaciones más eficientes en problemas de imágenes médicas, espectroscopia, comunicaciones ópticas, astronomía, química y biología molecular.

Areas de investigación importantes son llegar dentro del marco de la decisión cuántica a los procesos estocásticos, control optimal y teoría de la información. Todas ellas proyectaran luz nueva tanto sobre la física cuántica, como sobre la decisión bayesiana.

b, 11) Teoría de Juegos y Biología evolutiva

Las ideas originales de John Maynard Smith (1982), que se vienen a implicar en el nuevo concepto de estrategia evolutiva estable y la idea de equilibrio estratégico de la teoría de juegos, fueron el estímulo para la organización del Coloquio de Bielefeld en 1985, por el Premio Nobel Prof. Selten.

Entre las importantes discusiones de biólogos, economistas y teóricos de juegos, sobre los variados temas del coloquio recordemos como ejemplo típico la comunicación de Regelmann y Curio titulada “ Tit for tat in the great tit", que estudia experimentalmente la estrategia Tit for Tat, que utiliza el pájaro llamado gran Tit para proteger su prole de los predadores, y que es la misma del famoso dilema de los presos (Axelrod, 1984). No deja de ser interesante observar que muchos animales, y aún plantas, se ajustan en sus comportamientos incluso mas a la ley de maximización de la utilidad esperada que los humanos. Por ejemplo, en ratas se ha observado una mejor predicción que en los seres humanos de los resultados (premiados cuando hay acierto) de una sucesión de sorteos con dos resultados posibles $\mathrm{L}, \mathrm{R}$, de probabilidades $3 / 4,1 / 4$. Tras estas interesantes indicaciones de que la ley de M. U. S. E. tiene un carácter de universalidad como característica del comportamiento de los seres vivos, pasemos a los aspectos teóricos.

Se puede decir que una nueva "teoría evolutiva de juegos" está surgiendo a partir de los ochenta, estimulada por problemas biológicos que dan lugar a modelos secuenciales de conflictos competitivos 


\section{Sixto Ríos}

y cooperativos en ambiente aleatorio en la que se apoyan otras nuevas disciplinas como las teorías de la información y la comunicación, la mejora de las soluciones secuenciales por aprendizaje... Tras su éxito en Biología Genética (Maynard, Smith, Axelrod, Schuster, Friedman,...) ha encontrado importantes aplicaciones en conflictos de la economía, medio ambiente, culturales, éticos, políticos,... Dentro de esta teoría general de la evolución, la "adaptación Darwiniana" se sustituye por sucesiones de soluciones óptimas que conducen a formas sociales relativamente estables. Así, la selección vuelve a ser influenciada por sucesos aleatorios y la memoria individual y colectiva y la transmisión de soluciones de conflictos precedentes, conectan el pasado con el presente y permiten el aprendizaje intergeneracional. En ellos juega un papel importante la actitud frente al riesgo a través de la regulación de la utilidad esperada que valen no solo para humanos, sino también para poblaciones animales.

b, 12) Teoría de patrones, decisión bayesiana y percepción visual con ordenador

El análisis bayesiano de decisiones, y la teoría de patrones han contribuido decisivamente a partir de 1984 al desarrollo de metodologías importantes para el problema de la modelización de la percepción sensorial, especialmente de la visión humana con ordenador y el reconocimiento del lenguaje hablado o escrito.

Una mención especial hay que hacer de los trabajos profundos de U. Grenander que inició hace más de treinta años una importante "teoría de patrones", con lo que ha creado una fecunda escuela, sobre todo a partir de 1993 en que se publica su libro, General Pattern Theory de más de mil páginas de matemática teórico-experimental.

Pronto se advierte la importancia de la consideración de los procesos de análisis y de síntesis a partir de los elementos básicos que son los que Grenander llama generadores (g) considerados como imágenes abstractas de los patrones; pero la regularidad en su teoría es muy relajada de acuerdo con las necesidades de las aplicaciones biológicas en que hay que incluir también la conexión con el observador a través de la percepción visual, sensorial,...

Más concretamente la teoría de patrones se construye mediante conjuntos $(G)$ de objetos $g \in G$ entre los que se definen ciertas relaciones. Objetos o generadores organizados en una jerarquía de niveles, mientras las operaciones se relacionan a un álgebra universal definida en el conjunto $\mathrm{G}$ de elementos $\mathrm{g}$ álgebras parciales universales (en que algunas relaciones quedan parcialmente no definidas). Para completar el formalismo 


\section{Racionalidad y toma de decisiones hacia el siglo XXI}

considera también relaciones de conexión entre elementos $\mathrm{g} \in \mathrm{G}$ mediante grafos,.... .

De un modo genérico se puede decir que el problema perceptual que se le presenta a un observador se caracteriza por a) las inferencias que el observador hace de las formas del mundo exterior a partir de sus observaciones y b) la información que tales imágenes dan respecto de aquellas propiedades, que están determinadas por la estructura a priori del mundo.

La idea básica de la modelización bayesiana del problema de la percepción visual es caracterizar, como una distribución de probabilidad, la información relativa a una imagen de una escena vista en diferentes estados dados los datos correspondientes a las diferentes imágenes. Entonces la regla de Bayes suministra la herramienta fundamental de cálculo para pasar de estos dos factores a la distribución a posteriori. Y éste es esencialmente el esquema básico del enfoque del importante problema de la percepción, cuya solución final tanto interesa a la humanidad.

b, 13) Inteligencia artificial y sistemas expertos Vamos a referirnos brevemente a la relación del Análisis de Decisiones con los sistemas expertos, que utilizan los métodos de la Inteligencia Artificial para la toma de decisiones. Es bien sabido que los sistemas expertos tradicionales suministraban un soporte para las decisiones mimificando las recomendaciones de los expertos humanos y tratando la incertidumbre mediante métodos ad hoc, que no tienen en cuenta las relaciones de relevancia de los sucesos inciertos.

Con el nuevo enfoque probabilístico se trata de establecer una sucesión de dependencias que permitan tener en cuenta en los sistemas de Inteligencia Artificial las relaciones primitivas que se designan como verosimilitud comparada, condicionamiento, relevancia, causación,..., que adecuadamente establecidas permiten utilizar la inferencia bayesiana para llegar a las probabilidades de las complejas proposiciones finales. Este estudio, que ha obligado ha profundizar la axiomática de las dependencias ha originado la introducción de los grafos bayesianos, diagramas de influencia, etc. Ellos permiten establecer un conjunto coherente de dependencias directas o indirectas que constituyen la esencia cualitativa de los modelos de bases de conocimientos y de los sistemas expertos asociados a los problemas de decisión.

Recientemente, en 1990, ha construido Heckerman un sistema experto llamado normativo, que mejora notablemente la precisión en el tratamiento de la incertidumbre, apoyándose en la regla de la esperanza de utilidad, llamada por él "regla de oro" de la decisión. Mediante los nuevos conceptos de similaridad y partición asociados a la representación de la incertidumbre en el llamado grafo de creencias, logra una simplificación 


\section{Sixto Ríos}

464

en el cálculo de probabilidades subjetivas. En el experto que llama Pathfinder, dedicado al tratamiento de 60 enfermedades que se presentan en los ganglios linfáticos, logra reducir de 75000 a 14000 el número de probabilidades que se han de obtener para su construcción. Es, en definitiva, a parte de la importancia de su aplicación concreta, una prueba de que la identificación de formas específicas de independencia condicional y la utilización de las mismas en la representación de conocimientos, puede permitir la construcción de sistemas expertos normativos válidos para decisiones de gran complejidad.

La discusión entre teorías normativas y descriptivas equivaldría ahora a considerar el problema de si, por ejemplo, un medico debe tomar sus decisiones ayudado o no por, el análisis de decisiones.

Como dice Heckerman, los sistemas expertos normativos, es decir, sistemas expertos que sitúan el conocimiento de expertos en el marco del análisis de decisiones, tienen la posibilidad de incrementar la calidad de las decisiones tomadas por los médicos y de mejorar de una forma dramática el resultado del paciente. Tales metodologías se aplican actualmente al problema de la ictericia del recién nacido por el GAD de la $\mathrm{F}$. de Informática asociado al GAD del Hospital G. Marañón y al GAD de la R. Academia de Ciencias.

Una de las propiedades de un sistema experto debe ser tener la facultad de aprender y mejorar observando y analizando los problemas que puede resolver. El sistema va adquiriendo así habilidad para resolver problemas no idénticos a los que ha resuelto anteriormente. Se suelen considerar varios problemas de aprendizaje: aprendizaje supervisado, análogo al de un niño guiado por su padre o maestro que le instruye en una tarea específica; aprendizaje no supervisado, en que el sistema aprende de sus propias experiencias intentando soluciones o mediante observación pasiva. Un tercer tipo llamado instrucción selectiva es una mixtura de aquellos. La meta es que el sistema de aprendizaje no solo sea capaz de adquirir nuevo conocimiento, sino de perfeccionar su proceso de razonamiento y reordenar su conocimiento en estructuras más eficientes. Este ambicioso programa (Koldner, 1984) está atrayendo un gran número de investigaciones teóricas y de laboratorio. Por ejemplo, en el libro de Patrick se presenta un nuevo aspecto de aprendizaje no supervisado mediante una generalización del teorema de Bayes realizada por el autor. Como en el teorema clásico de Bayes las categorías son mutuamente exclusivas, pero pueden ser, estadísticamente dependientes. Este nuevo enfoque permite tratar clases complejas como, por ejemplo, enfermedades múltiples simultáneas en el mismo paciente. Concretamente la aplicación de la metodología estadística a establecer las ventajas de unos métodos de aprendizaje respecto de otros es un problema actualmente en sus 


\section{Racionalidad y toma de decisiones hacia el siglo XXI}

comienzos. Es una faceta de enfoque más amplio de colaboración de la Estadística con la Inteligencia Artificial en el estudio y comparación de la eficacia de las máquinas inteligentes.

Finalmente es interesante indicar el papel de la Inteligencia artificial como motor de impulsión de importantes investigaciones estadísticas actuales, entre las que destacamos el análisis exploratorio de datos, la modelización, el metanálisis, minería de datos,...

En el primer campo está el estudio de las implicaciones de los métodos de reconocimiento de formas desarrollados en el marco de la inteligencia artificial.

En el segundo se consideran sistemas complejos de inferencia que permiten tratar problemas estadísticos complejos con técnicas similares a las que se desarrollan en los sistemas expertos.

En el tercero se logra una síntesis de múltiples análisis de datos relativos a un mismo problema lográndose una mejora efectiva en la solución.

En el cuarto se trata de la exploración de grandes bancos de datos para extraer patrones e imágenes.

Vemos, pues, que hay aquí un dominio de trabajo interdisciplinar que requiere la colaboración de probabilistas, informáticos, médicos, geólogos, abogados, economistas,...

\section{Epílogo}

En estos penúltimos años de mi actividad científica he emprendido algunos trabajos en la Real Academia de Ciencias, gracias especialmente a la eficaz y competente colaboración de varios compañeros.

Espero que por los años que me quedan de lucidez intelectual pueda continuar colaborando en la revolución probabilística.

Concretamente, hemos constituido una Federación de Grupos de Análisis de decisiones resultante de la colaboración del Grupo de Análisis de Decisión (GAD) de la Real Academia de Ciencias con otros de varias Universidades, Escuelas Técnicas Superiores, Hospital Gregorio Marañón, Ministerio de Defensa,...

Se han realizado ya trabajos de interés para nuestro país, gracias a Ayudas de la Fundación BBV, Comunidad de Madrid, Instituto de España,... Se han publicado tres Volúmenes de Coloquios Internacionales y varios nacionales, $\ldots$

Esperamos y creemos que con estas armas podremos seguir ayudando al desarrollo científico-social de nuestro país en el siglo XXI.

Ahora que estamos una vez más en vísperas de reformas en la enseñanza valgan para empezar, estos primeros flechazos: los avances ac- 
tuales del Análisis de decisiones en relación no ya con la investigación científica y el desarrollo, sino con la práctica diaria actualmente correcta de profesiones como médicos, abogados, economistas, psicólogos, políti$\cos$, militares,... en las que continuamente han de tomarse decisiones en incertidumbre se hace fundamental avanzar y salir de la "época de la media y la varianza" y poner al día (como en algunos países) la cultura probabilística o de la incertidumbre no solo de los profesionales sino de la sociedad culta que es afectada por sus decisiones, para que las acoja y comprenda con respeto y entusiasmo.

Los psicólogos Kahnemann y Tversky han dicho que la mente humana presenta una laguna importante: "la gente no suele seguir el cálculo de azar o la teoría de la predicción estadística, pues por alguna razón nuestras mentes no están construidas para trabajar con las reglas de la probabilidad".

Pensamos que quizá la explicación está en el importante lapso de tiempo que la mente humana necesita normalmente para habituarse a una nueva forma de pensar que implique en nuestros comportamientos el cálculo de probabilidades. Hay que tener en cuenta que así como los razonamientos de la lógica deductiva y los comportamientos humanos asociados se vienen practicando al menos desde Aristóteles, la lógica inductiva no se inició hasta que Laplace (1812) redescubrió el teorema de Bayes.

Gracias finalmente a mi nieta Elisa por su ayuda eficaz con el ordenador.

\section{Referencias}

Arrow, K.J. (1951), Social Choice and Individual Values, Yale Univ. Press. Axelros, R. (1984), The Evolution of Cooperation, Basic Books, New York.

BAYES, T. (1763), "An Essay Towards Solving a Problem in the Doctrine of Chance", with Richard Price's foreword and discussion, Phil. Trans. Roy. Soc, 370-418.

BERger, J.- Ríos, D. (1997) Recente Developments in Bayesian Inference with applications in Hydrology. Advances in Stochastic Hydrology. UNESCO.

Bernoullu, J. (1713), Ars Conjectandi, Impensi Thurnisiorum: Fratum, Basilea.

Bernouilli, D. (1738), "Specimen Theoriae Novae de Mensura Sortis" Commentarii Academiae Scientiae Imp. Ptropolitanae, V, 175-192.

Condorcet, M.J.A.C. (1785), Essai sur l'Application de l'Analysea a la Probabilité des Décisions Rendues a la Pluralité des Voix, Paris.

DebreU, G. (1954), "Representation of a Preference Ordering by a Numerical Function” en Decision Processes, R.M. Tharll, C.H. Coombs y R.L. Davies (eds), Wiley, New York.

De Finetri, B. (1931), Probabilismo, Saggio Critico sulla Teoría delle Probabilitá e sul Valore della Scienza, F. Perella, Naples. 


\section{Racionalidad y toma de decisiones hacia el siglo XXI}

Fishburn, P.C. (1982), The Foundations of Expeted Utility, Reidel Dordrecht, The Netherlands.

FISHBURN, Wakker, (1995) "Inventoin of independence condition for preferences". Management Science Vol. 41,1130-1144

FrISCH, R. (1982), "Cooperation Between Politicians and Econometricians on the Formalization of Political Reference", Reunión de Premios Nobel en Lindau.

HACKING, I. (1991), The Emergence of Probability, Cambridge University Press.

Heckerman, D. (1991), Toward normative expert Systems: The Pathfinder Project, Cambrige U.P.

HowARD, R. (1964), The Principles and Applications of Decision Analisys, Strategic Decision Group, Menlo Park, California.

KEENEY, R. y H. Raiffa (1976), Multiobjective Decision Making, Wiley, New York.

MAYNARD-Smith, J. (1982), Evolution and the Theory of Games, Cambridge U.P.

Prelec and LOWenstein, (1991), "Decision Making over Time and under uncertainty". Manag. Science, vol. 37

RAIFfa, H. (1968), Decision Analisys, Addison Wesley, Reading Mass.

RaIfFA, H. (1982), The Art \& Science of Negotiation, Harvard U.P., Cambrige Mass.

Rios, S. (1967) Procesos Dinámicos de Decision, Memorias de la Academia de Ciencias, Madrid.

Rios, S. (1995), Modelizacion, Alianza Editorial, Madrid.

Rís-InsuA, D. (1992), Sensitivity Analysis in Multiobjective Decision Analysis. Springer Verlag.

RIos, S. (1995), "Hacia el Siglo XXI: Las Decisiones "Discurso de Ingreso como Académico de Honor, Real Academia Sevillana de Ciencias, Sevilla.

Rios - InSUA, D. (1995), The Operation on Kariba Lake, Multicriteria Decision Analy. sis.

Rios, S., Rios - Insua, S., Rios - Insua D., G. Barreno, P., Allais Phenomena and Completenes of Preferences (Act. FUR VI).

Rfos, S., Rfos - Insua M.J., Rfos - Insua, S. (1989), Procesos de Decision Multicriterio, EUDEMA, Madrid.

SAVAGe, L.J. (1954), The Foundations of Statistics, Wiley, New York.

Simon, H.A., Dantzig, G.B., Raiffa, H. (1992), Decision Making and Problem Solving (en Decision Making Ed. Mary Zey. SAGE Publications).

Von Neumann, J. y MoRgenstern, O. (1944), Theory of Games and Economic Beha. viour, Princeton University Press, Princeton.

WaLD, A. (1945), "Statistical Decision Functions wich Minimize the Maximum Risk", Ann. Math. 46.

WaLd, A. (1950), Statistical Decision Functions, Wiley, New York. 